\title{
Observable interactions of quintessence with ordinary matter and neutrinos
}

\author{
R. Horvat \\ "Rudjer Bošković" Institute, P.O.Box 180, 10002 Zagreb, Croatia
}

\begin{abstract}
For any realistic theory of quintessence that allows for a violation of the equivalence principle (VEP), we study the experimental constraints on such theories coming from ordinary matter as well as neutrinos. We discuss and compare constraints from two basically different (extra) contributions to differences of fermion masses: one is due to the vacuum expectation value (vev) of quintessence, and the other is based on a quintessence-exchange graph at finite temperature/density.

98.80.Cq, 95.35.+d
\end{abstract}

Typeset using REVTEX 
There are now increasing indications, based on recent redshift-distance measurements of High-Z Supernovae Ia [四, measurements of different observables on rich clusters of galaxies which all point towards a low value of the fraction density in matter [2] as well as recent Cosmic Microwave Background measurements [3], that the Universe is presently undergoing accelerated expansion due to a smooth component with negative pressure, sometimes also called 'dark energy' 叫. For a long time the simplest candidate has been a nonvanishing cosmological constant. Such a situation has changed recently, when a dynamical, slowlyrolling, spatially inhomogeneous scalar field component, named 'quintessence' [5], had been put forward as an alternative candidate. Generally, models of quintessence are currently better motivated; because of more parameters involved in such models they have proven more useful in explaining some of the difficulties left by the cosmological constant scenarios. Besides the 'cosmological constant problem' [6], the quintessence models face the 'why now' problem: since the dark energy density and the matter energy density decrease at different rates as the Universe expands, it is not clear why we appear to live in an era during which the two energy densities are roughly the same, with the concordant values, $\Omega_{\Lambda} \sim 2 / 3, \Omega_{m} \sim 1 / 3$ [7], $\Omega_{m}$ and $\Omega_{\Lambda}$ being the fraction densities in matter and cosmological constant, respectively.

In order to deal at least partially with the fine-tuning problems stated above, typically quintessence models possess a special sort of scalar potential, 'tracking attractor solutions' [8], whose cosmology is the same and independent of a wide set of initial conditions (around 100 orders of magnitude). This means that the scalar field energy density at present can be reached starting from a large range of initial conditions. Undoubtedly this fact has given an impetus for further development of quintessence models.

In spite of such a progress, it turns out that the quintessence idea is difficult to implement in the context of realistic models [9,[0]. The main problem besetting the quintessential scenario has to do with the following two facts: (a) the quintessence field should be very light, (b) time dependence of the expectation value of the field $\phi$, of order $M_{p}$ [ $\mathbb{\|}\left(M_{p}=\right.$ $2.4 \times 10^{18} \mathrm{GeV}$ is the reduced Planck scale ) at present, is related to a phenomenon of the time variation of the constants of nature. In the former case, Eötvös-type experiments put severe 
constraints on the couplings of quintessence with ordinary matter [9]. The quintessenceexchange forces would otherwise become observable. A phenomenon related to the case (a) is that the same couplings would unavoidably generate higher-order corrections in the quintessence potential, thereby spoiling the standard flatness conditions for $V(\phi)$ [10].

Specifically, a coupling $\beta_{G^{2}}\left(\phi / M_{p}\right) \operatorname{Tr}\left(G_{\mu \nu} G^{\mu \nu}\right)$, where $G_{\mu \nu}$ is the field strength tensor for QCD, is strongly constrained [9] by Eötvös-type experiments,

$$
\left|\beta_{G^{2}}\right| \leq 10^{-4}
$$

Similarly, a coupling such as $\beta_{F^{2}}\left(\phi / M_{p}\right) F_{\mu \nu} F^{\mu \nu}$ will lead to the evolution of the fine-structure constant $\alpha$. Since the present-day value for $\langle\phi\rangle$ is of order $M_{p}$ 円, this easily might generate corrections of order one to the gauge coupling. On the other hand, as the time dependence of the fine-structure constant is very strongly constrained [11], a limit [9]

$$
\left|\beta_{F^{2}}\right| \leq 10^{-6} \frac{M_{p} H_{0}}{<\dot{\phi}>}
$$

can be obtained, where $H_{0}$ is the present value of the expansion rate and $\langle\dot{\phi}\rangle$ is the average value over the last two billion years.

Note that (1) and (2) actually represent a moderate fine-tuning in a theory. Indeed, from a traditional viewpoint, the expected values for $\beta$ s are of order unity, since they represent interactions at the Planck scale. It should be stressed that it is possible to alleviate the above problem by imposing an approximate global symmetry, but this works only in pseudo-Goldstone models of quintessence [9]. A solution to the problem in the form of the 'least coupling principle' of Damour and Polyakov [13] in string theory has recently been proposed [12]. Such a principle was originally formulated for the string dilaton field [or any other gauge-neutral massless scalar field (moduli) present in string theory]. By accepting the 'least coupling principle' here, we must assume that the mechanism is also operative

\footnotetext{
${ }^{1}$ In the following, we shall always consider scalar field based quintessence models belonging to the class for which $<\phi_{0}>\sim \mathcal{O}\left(M_{p}\right)$, where the subscript ' 0 ' denotes the present-day value.
} 
for quintessence since a recent analysis [14 shows that the dilaton with an exponentially decreasing potential cannot provide us with the negative equation of state, neither in the radiation-dominated nor matter-dominated era. Hence, the dilaton is useless for the dynamical component of quintessence [note that Refs. [12] do not aim to prove the 'least coupling principle' for quintessence, but rather to propose a solution much in the spirit of the 'least coupling principle' of Damour and Polyakov]. Nevertheless, observational constraints on cosmological models with quintessence arising from moduli fields have recently been studied [15], with the conclusion that for parameter values away from the attractor there can exist models which are consistent with the observational tests.

As mentioned earlier, $<\phi_{0}>\sim \mathcal{O}\left(M_{p}\right)$. This means that we need to consider the full functions, e.g.

$$
\beta_{F^{2}}\left(<\phi_{0}>\right) \equiv \sum_{n=0}^{\infty} \beta_{F^{2}}^{n}\left(\frac{<\phi_{0}>}{M_{p}}\right)^{n}
$$

and similarly for other $\beta$ s. The 'least coupling principle' states that string-loops effects must generate a non-trivial dependence of the coupling functions entering the effective Lagrangian [like that in Eq.(3)], in order to admit extrema at finite values of the $\phi$ 's vev. Specifically, the mechanism provides that a coupling of the scalar (flavor 'independence' coupling of the string dilaton field in the scenario of Damour and Polyakov) with the rest of the world has a common minimum close to the present value of the $\phi$ 's vev, and that the cosmological evolution naturally drives the vev toward the minimum where the scalar decouples from matter. In addition, even if a strictly massless dilaton exists, the model is able to satisfy the high-precision test of the equivalence principle $\left(\sim 10^{-12}\right.$ level $)$, allowing the coupling constants that represent interactions at the Planck scale to be species-dependent, thereby violating the equivalence principle (VEP).

In the present paper, we study constraints from the sector of ordinary matter and neutrinos on the quintessential Yukawa couplings. We assume VEP and find that such theories can be constrained substantially in both sectors. We start by assuming that $\phi$ can couple to the standard-model fermions via interactions of the form 


$$
\beta_{f} \frac{\phi}{M_{p}} \mathcal{L}_{Y u k}^{f}
$$

where $\beta_{f}$ is a dimensionless coupling and $\mathcal{L}_{Y u k}^{f}$ is the gauge-invariant coupling of the Higgs doublet $\Phi$ with a standard-model fermion. When $S U(2) \times U(1) \rightarrow U(1)_{e m}$, the field $\Phi$ acquires a non-zero expectation value, $\langle\Phi>$, and the Yukawa couplings turn into

$$
\beta_{f} \frac{m_{f}}{M_{p}} \bar{\psi}_{f} \psi_{f} \phi
$$

Hence, these couplings can generate an extra Dirac mass term for the fermions. Let us stress that $\beta_{f}$ from (5) has been recently constrained for the sector of neutrinos. Indeed, we have recently shown [16 that by assuming an interaction of quintessence with the cosmic neutrino background (CNB), it is possible to obtain a limit on $\beta_{\nu}$

$$
\beta_{\nu} \lesssim 2 \times 10^{-3}\left(\frac{\mathrm{eV}}{m_{\nu}}\right)^{\frac{3}{2}} .
$$

The limit (6) obviously depends on neutrino mass and only for large neutrino masses, $m_{\nu} \sim$ $1 \mathrm{eV}$ f, Eq. (6) represents a moderate fine-tuning in $V(\phi)$. If we set $m_{\nu} \sim 0.05 \mathrm{eV}$ consistent with the Super-Kamiokande experiment [18], then $\beta_{\nu} \lesssim \mathcal{O}\left(10^{-1}-1\right)$. Obviously, we need neither suppression by some symmetry nor the 'least coupling principle' to achieve these values.

Next, by considering the experimental constraints from ordinary matter and assuming VEP, we show how stringent constraints on the quintessential Yukawa couplings can be obtained. One can read off from (5) the effective fermion mass as

$$
m_{i}^{e f f}=m_{i}+\beta_{i} \frac{<\phi_{0}>}{M_{p}} m_{i},
$$

where the species-dependent parameters $\beta_{i}$ characterizes VEP. Since $<\phi_{0}>\sim \mathcal{O}\left(M_{p}\right)$, the induced mass in (7) can be as large as the bare mass if $\beta_{i}$ are of order unity. Again, we have to consider the full functions $\beta_{i}\left(<\phi_{0}>\right)$, so that the present effective mass $(7)$ reads

\footnotetext{
${ }^{2} \mathrm{In} \Lambda \mathrm{CDM}$ models, the presence of hot dark matter is no longer necessary and therefore $\mathrm{eV}$ neutrinos are not needed to provide this component [17].
} 


$$
m_{i}^{e f f}\left(<\phi_{0}>\right)=m_{i}+\beta_{i}\left(<\phi_{0}>\right) m_{i} .
$$

If the 'least coupling principle' is at work, then $\beta_{i}\left(<\phi_{0}>\right)$ can stay very close to the minimum value, and hence $\beta_{i}$ can be much less than unity.

Now, we apply the mechanism (8) to constrain VEP from the experimental constraint on the $K_{L}-K_{S}$ mass difference. By noting from (8) that the gravitational and the mass eigenstates are identical, the effective $K_{L}-K_{S}$ mass difference now reads

$$
m_{L}^{e f f}\left(<\phi_{0}>\right)-m_{S}^{e f f}\left(<\phi_{0}>\right)=m_{L}-m_{S}+m_{L}\left[\beta_{L}\left(<\phi_{0}>\right)-\frac{m_{S}}{m_{L}} \beta_{S}\left(<\phi_{0}>\right)\right] .
$$

Using the experimental value $\left(m_{L}-m_{S}\right) / m_{L} \sim 7 \times 10^{-15}$ [19], taking an agreement of exact calculations of the mass difference with experiment into account, and setting $m_{S} \simeq m_{L}$ we get a stringent limit

$$
\Delta \beta_{L, K}<7 \times 10^{-15}
$$

where $\Delta \beta_{L, K} \equiv \beta_{L}\left(<\phi_{0}>\right)-\beta_{S}\left(<\phi_{0}>\right)$ now characterizes VEP. It is very important to note that this bound is even better than the most severe limit $\left(\sim 10^{-12}[20]\right)$ obtained from the extremely stringent tests of the equivalence principle. The above bound being independent of the absolute values of $\beta_{L}$ and $\beta_{S}$ is relevant even if we were to invoke a solution in the spirit of the 'least coupling principle' of Damour and Polyakov (with $\beta_{L, S} \ll 1$ ). Finally, we would like to stress that differences of the couplings to composite objects as in (10) are expected to contain, on the QCD basis alone, extra small parameters in the form of the ratio of the quark masses to the hadron mass, or the fine-structure constant. They cannot, however, be solely responsible for the very small number in (10).

Let us now discuss a special distinction between the "bare" and the "effective" quantities entering (7) and (8), and a way to detect such a shift as well. Since we consider here the non-universal couplings of quintessence in the "effective" part of (7) and (8), they apparently induce violation of the universality of free fall. Hence, we actually consider the compositiondependence of the quintessential coupling, i.e. its dependence on the type of matter under 
consideration. In such a case it can be easily shown 13 that, in the gravitational field generated by some external mass, two test bodies will fall with acceleration difference proportional to the difference in the couplings defined as in (5). Similarly, the additional term in the free fall acceleration of a body in the gravitational field of the Earth will be proportional to the spacetime gradient of its effective mass. This spacetime dependence can be clearly seen from (8). The $\phi$-dependence of the $\beta$ 's entails a corresponding $\phi$-dependence, and therefore a spacetime dependence of $m_{i}^{e f f}$. Since different bodies $\left(K_{L}, K_{S}\right)$ have different contributions to their $m_{i}^{e f f}$, we expect the acceleration difference to differ from zero.

Giving attention to another (predominately) smoothly distributed component in the Universe, the CNB, we now describe how sizeable fermion masses can be generated in the matter. From a viewpoint of Thermal Field Theory (TFT), fermion masses within the CNB can be generated via a quintessence-exchange tadpole graph. As a constant independent of the external fermion momentum, the tadpole graph at finite temperature/density is directly related to fermion mass. It consists of a medium-induced loop (in which real relic neutrinos are circulating) and also of a one-loop resummed propagator for the scalar. Using the real-time version of TFT [21], we find by explicit calculation a contribution to the induced fermion mass as

$$
m_{f}^{i n d} \simeq 0.26\left(\beta_{\nu} m_{\nu} T_{\nu}^{3}\right)\left(\beta_{f} m_{f}\right)\left(m_{\phi}^{b} M_{p}\right)^{-2}
$$

where $T_{\nu}$ and $m_{\nu}$ are the relic neutrino temperature and the heaviest mass from the CNB, respectively. Here $\beta_{f, \nu} \equiv \partial \ln \beta_{f, \nu}(<\phi>) /\left.\partial\left(<\phi>/ M_{p}\right)\right|_{<\phi>=<\phi_{0}>}$ measures the strength of the coupling of quintessence to the $f, \nu$-particles. Eq. (11) assumes that the bare mass for $\phi\left(m_{\phi}^{b} \equiv \sqrt{V^{\prime \prime}\left(<\phi_{0}>\right)}\right)$ is always larger than the quintessential thermal mass. This is justifiably as in order for our epoch to correspond to the beginning of slow-rolling regime, one should require that the effective mass of fluctuations in $\phi, \sqrt{V^{\prime \prime}\left(<\phi_{0}>\right)} \simeq H_{0}$. On the other hand, the thermal mass for $\phi$ can be $m_{\phi}^{t h} \leq H_{0}$. The properties of such graphs, but where the thermal mass dominates over the bare mass, were discussed first time in the context of the dilaton-exchange gravity in 22$]$. 
Next, by combining the constraint from the neutrino sector (6), with Eq. (11), we obtain an upper limit on $\Delta \beta_{L, K}$

$$
\Delta \beta_{L, K}<1.4 \times 10^{-11}\left(\frac{1.7 \times 10^{-4} \mathrm{eV}}{T_{\nu}}\right)^{3}\left(\frac{m_{\nu}}{0.05 \mathrm{eV}}\right)^{\frac{1}{2}}
$$

where we have used the experimental constraint on the $K_{L}-K_{S}$ mass difference. In (12) we have put for $\beta_{\nu}$ a half of the limiting value (6), since $m_{\phi}^{b} \simeq m_{\phi}^{\text {th }}$ near the limit. The bound (12) becomes weaker when $\beta_{\nu}$ is decreasing. Note that because of the negative sign of the square of $m_{\phi}^{\text {th }}$ for $T_{\nu} \ll m_{\nu}$ (first ref. in [12]), a more sophisticated treatment is needed when $m_{\phi}^{b} \simeq m_{\phi}^{t h}$. Obviously, the best bound in (12) is achieved when neutrino masses are hierarchal with the highest value of about $0.05 \mathrm{eV}$, as indicated by the Super-Kamiokande result. Here we stress that the effect of neutrino clustering, which is not included in (12), would lead to a somewhat stronger constraint. Besides, in the case when a large neutrino asymmetry exists, the bound can be improved by about two orders of magnitude with respect to the non-degenerate case (12). Nevertheless, the bound (10) obtained from the present-day value of the $\phi$ 's vev, remains superior by a few orders of magnitude.

Next, consider the sector of neutrinos. In the context of neutrino oscillation solution to the existing neutrino anomalies, the best constraints are expected to come from that solution with the least mass squared difference. Hence, the obvious candidate is an explanation of the solar neutrino data via neutrino oscillation in vacuum, with $\Delta m^{2} \simeq 7.5 \times 10^{-11} \mathrm{eV}$ [23]. Note that VEP will be most prominent if the oscillatory neutrinos are completely degenerate. P Then, by combining Eqs. (7) and (6) we derive $\Delta m^{2}$ for two degenerate-in-mass neutrinos with $m \sim 1 \mathrm{eV}$ as

$$
\Delta m^{2} \simeq 2 m^{2} \Delta \beta_{\nu}
$$

where $\Delta \beta_{\nu} \equiv \beta_{\nu_{2}}-\beta_{\nu_{1}}$. For lighter neutrinos, the bound (6) is no longer restrictive and an extra term, $m^{2}\left(\beta_{\nu_{1}}+\beta_{\nu_{2}}\right) \Delta \beta_{\nu}$, can be of the same order of magnitude as (13). However,

\footnotetext{
${ }^{3}$ The degeneracy can be protected by a presumed global inter-family (flavor or horizontal) symmetry of leptons.
} 
in that case the bound on $\Delta \beta_{\nu}$ is weaker. One can thus conclude that the current solar neutrino data can probe VEP at the level

$$
\Delta \beta_{\nu} \simeq 10^{-10}\left(\frac{\mathrm{eV}}{m}\right)^{2} .
$$

Note that the limit (14) is much better than the limit [24] obtained by comparing neutrinos and antineutrinos from SN 1987A. The corresponding limit on $\Delta \beta_{\nu}$ obtained from the tadpole graph is always much weaker, irrespective of the neutrino mass.

Finally, we discuss constraints from the neutrinoless nuclear double beta decay $(0 \nu \beta \beta$ decay; for review see [25]). For that purpose we need the (1,1) entry of the neutrino mass matrix in the weak basis. For two flavors we easily obtain

$$
M_{\nu}^{e e}=\frac{m_{\nu_{1}}^{e f f}+m_{\nu_{2}}^{e f f}}{2}+\frac{1}{2}\left(m_{\nu_{1}}^{e f f}-m_{\nu_{2}}^{e f f}\right) \cos 2 \theta .
$$

Here, the neutrino weak interaction (flavor) eigenstates are assumed to be linear superpositions of the mass eigenstates with a mixing angle $\theta$. Assuming two degenerate neutrinos, the VEP part in (15) reads $\frac{1}{2} m \Delta \beta_{\nu} \cos 2 \theta$. We see that this contribution is very similar to that in (13). Since the bounds from the present $0 \nu \beta \beta$-decay experiments $\left(M_{\nu}^{e e}<0.2 \mathrm{eV}[26]\right)$ are much less stringent than those from neutrino oscillation experiments, one may conclude that the bound on $\Delta \beta_{\nu}$ from the $0 \nu \beta \beta$-decay is much weaker than those given by the above expressions.

To summarize, we have found interesting new bounds for possible contributions of quintessence to VEP. We have applied the experimental constraints, both from the sector of ordinary matter and neutrinos, on some quintessence-induced contributions to fermion masses. In some cases our bounds are better than the upper limits from present experimental data on the universality of the free fall. Our bounds are always better than the corresponding supernova limits.

Acknowledgments. The author acknowledges the support of the Croatian Ministry of Science and Technology under the contract 0098011. 


\section{REFERENCES}

[1] S. Pearlmutter at al., Nature (London) 391, 51 (1998); A. G. Riess et al., Astron. J. 116, 1009 (1998).

[2] S. D. M. White, J. F. Navarro, A. E. Evrard and C. S. Frenk, Nature (London) 366, $429(1993)$.

[3] P. de Bernardis at al., Nature (London) 404, 955 (2000).

[4] M. S. Turner, Nucl. Phys. Proc. Suppl. 72, 69 (1999).

[5] R. R. Caldwell, R. Dave and P. J. Steinhardt, Phys. Rev. Lett. 80, 1586 (1998).

[6] For a review see S. Weinberg, Rev. Mod. Phys. 61, 1 (1989).

[7] see e.g. ref.[4].

[8] I. Zlatev, L. Wang and P. J. Steinhardt, Phys. Rev. Lett. 82, 896 (1999); P. J. Steinhardt, L. Wang and I. Zlatev, Phys.Rev. D59 123504 (1999).

[9] S. M. Carroll, Phys. Rev. Lett. 81, 3067 (1998).

[10] C. Kolda and D. Lyth, Phys. Lett. B458, 197 (1999).

[11] T. Damour and F. Dyson, Nucl. Phys. B480, 37 (1996).

[12] R. Horvat, Mod. Phys. Lett. A14, 2245 (1999); A. Masiero, M. Pietroni and F. Rossati, Phys. Rev. D61, 023504 (2000).

[13] T. Damour and A. M. Polyakov, Gen. Rel. Grav. 26, 1171 (1994); Nucl. Phys. B 423, 532 (1994).

[14] P. Binetruy, Phys. Rev. D60, 063502 (1999).

[15] S. C. C. Ng, astro-ph/0004196.

[16] first reference in [12]. 
[17] Relic Neutrino Workshop, Sep 98, Trieste; A. Dighe, S. Pastor and A. Yu. Smirnov, hep-ph/9812244.

[18] (Super-Kamiokande coll.) K. Scholberg, hep-ex/9905016.

[19] Review of Particle Properties, C. Caso et al., European Physical Journal C3, 1 (1998).

[20] B. R. Heckel et al., Phys. Rev. Lett. 63, 2705 (1989); E. Adelberger et al., Phys. Rev. D 42, $3267(1990)$.

[21] A. J. Niemi and G. W. Semenoff, Nucl. Phys. B 230, 181 (1984); R. L. Kobes and G. W. Semenoff, Nucl. Phys. B 260, 714 (1985); Nucl. Phys. B 272, 329 (1986).

[22] R. Horvat, Phys. Rev. D58, 125020 (1998).

[23] For a recent review see e.g. V. Barger and K. Whisnant, hep-ph/0006235.

[24] M. J. Longo, Phys. Rev. Lett. 60, 173 (1988); L. M. Krauss and S. Tremaine, Phys. Rev. Lett. 60, 170 (1988); J. M. LoSecco, Phys. Rev. D 38, 3313 (1988); S. Pakvasa, W. Simmons and T. J. Weiler, Phys. Rev. D 39, 1761 (1989).

[25] H. V. Klapdor-Kleingrothaus, Springer Tracts in Modern Physics 163, 69 (2000).

[26] L. Baudis et al., Phys. Rev. Lett. 83, 411 (1999). 